\title{
Likhang - Modelo na 21st century skills development ladder gamit ang video production bilang tranpormatibong estratehiya
}

\author{
Galdo, Mark
}

Southern Leyte State University - Tomas Oppus, Philippines (mgaldo05@gmail.com)

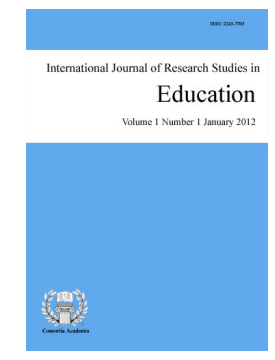

ISSN: 2243-7703

OPEN ACCESS DOI: $10.5861 /$ ijrse.2022.201

\section{Abstract}

A proof that 21st century practice is the subject of the teaching-learning process of the Philippine educational reform. Therefore, this paper is a contribution to the modern teaching-learning of millennia in the 21 st century with the help of technology. The paper focuses on the 21st century's advanced skills of tertiary students using video production as a transformative strategy. Using descriptive-exploratory through checklists, field notes, and focused-group discussion, all 21st century skills have been developed, but communicative and collaborative skills are most enhanced in every stage of video production. It has been discovered that communicative skills are developed in the development and pre-production stages, while collaborative skills are developed in the production and post-production stages of video production. Critical thinking skills culminate at every stage. It is evident that focusing on critical thinking skills should be addressed by educators of 21 st century students. Creating critical thinking-based activities is expected in each teaching strategy so students are more prepared to enter the digital generation. Additionally, exposures to teachers in the increase of critical thinking activities are implicated in the teaching-learning process of each teacher and are guided by modern teaching methods and strategies.

Keywords: 21 st century skills, education, transformative strategy, video production 


\section{Likhang - Modelo na 21st century skills development ladder gamit ang video production bilang tranpormatibong estratehiya}

\section{Introduksyon}

Sa pagpasok ng $21^{\text {st }}$ century, panibagong hamon sa pagbabagong -anyo ng proseso ng pagtuturo-pagkatuto ang makaagapay sa mabilis na hatak ng teknolohiya bilang paghahanda sa mga mag-aaral na maging produktibo sa hinaharap. Kadikit nito ay ang pagsisimulang paggamit ng makabagong teknolohiya na nagdulot ng makabagong estilo sa pagkatuto para pag-ibayuhin ang ugnayan sa pagpapaunlad ng 21 st century na kasanayan upang magkaroon ng panibagong estratehiya sa pagkatuto sa pagpapalinang nito gamit ang video production (Norton at Hathaway, 2010).

Ang video production bilang transpormatibong estratehiya ay isang panibagong dulog sa integrasyon ng teknolohiya sa pagtuturo na mas nakikilala ang kalikasan at katangian ng mga kasanayan ng mag-aaral sa $21^{s t}$ century. Nagsilbing tagalantad ito ng buhay at karanasan ng mag-aaral sa pagpapatalas ng karunungan at pagpapalawak ng kanilang kasanayan sa pagbuo ng panibagong kahulugan at karanasan mula sa dating kaalaman na kinakailangan sa $21^{\text {st }}$ century skills (Alvermann, 2002).

Ang pagtamasa ng estratehiyang ito ay nagbigay ng malaking kalinangan sa apat na kasanayan ng 21 st century - komunikasyon, kolaborasyon, kritikal na pag-iisip at malikhain pag-iisip. Mula sa kaugnay na pag-aaral ng mga edukador, inilarawan na ang video production ay nagpapataas ng antas ng motibasyon ng mag-aaral sa pagbuo ng produksyon maging ang kasiglahan ng mag-aaral na makisama at makilahok sa produksyon (Hoffenberg at Handler, 2001); mahalaga din sa awtentikong pagkatuto pati na ang kritikal na pag-iisip ng mga mag-aaral (Kearney at Schuck, 2006), nanghihikayat sa mag-aaral na mapatalas pa lalo ang kanilang pag-unawa sa isang bagay habang bumubuo ng produksyon (Snelson, 2018); nagsisilbing pantulong sa pagtuturo at pagkatuto gamit ang teknolohiya (Bull at Bell, 2010); at nag-uugnay sa mga kapwa mag-aaral na maging malikhain at kritikal sa pagbubuo ng isang masining na gawain gamit ang teknolohiya (Cayari, 2015). Makikita sa gawaing ito ang kabuuang transpormasyon ng mag-aaral sa video production na siyang naging daan sa pag-unlad ng kasanayan ng mag-aaral sa 21 st century. Patunay ito na may malaking nalilinang na kasanayan ang estratehiyang ito sa mag-aaral upang maging handa sa anumang hamon sa 21 st century.

Isinaad nina Hofer at Swan (2005) sa kanilang pag-aaral na limitado ang pag-aaral sa video production sa antas tersyarya sapagkat karamihan sa pag-aaral tungkol dito ay pawang kuro-kuro ng mga edukador at mag-aaral sa sekundarya at elementarya, samantalang walang pagsusuring naganap sa antas tersyarya. Bagaman makakuha ng pag-aaral na may kaugnayan sa ibang kurso ngunit walang pag-aaral na nakalap na tumutuon sa mga mag-aaral sa tersyarya na kumukuha ng kursong pang-edukasyon.

Sa papel na ito, pinag-aaralan ng mananaliksik ang video production bilang transpormatibong estratehiya sa paglinang ng $21^{\text {st }}$ century skills sa antas tersyarya partikular sa mga mag-aaral sa kursong edukasyon. Sinuri ang mahahalagang komponents ng bawat kasanayan sa $21^{\text {st }}$ century (komunikatibo, kolaborasyon, kritikal na pag-iisip at pagkamalikhain) mula sa apat na yugto ng video production (development, pre-production, production, post-production) upang mailahad ang mga espesipikong kasanayan na nararapat na pagtuonan ng pansin gamit ang panibagong transpormatibong estratehiya.

\section{Teoretikal at Konseptwal na Balangkas}

Ang pag-aaral na ito ay nakasalig sa Tranformative Learning Theory ni Mezirow (2000) at sa ideya ni Dede (2010) sa konseptong pagpapalinang ng $21^{\text {st }}$ century na kasanayan sa mag-aaral.

Nakasaad sa Transformative Learning Theory ni Mezirow (2000) na ang mga makabuluhang konsepto ay maisalin sa bagong karanasan na maging isang kawili-wili. Bilang isang estratehiya, nakatutok ito sa mga 

mag-aaral na kinakailangang armasan ng mga pagpapahalaga at kasanayan na lilinang sa matalas na pananaw sa konkretong kalagayan ng daigdig at maghihikayat sa kanilang kumilos, indibidwal man o kolektibo upang paunlarin ang panlipunang kondisyon.

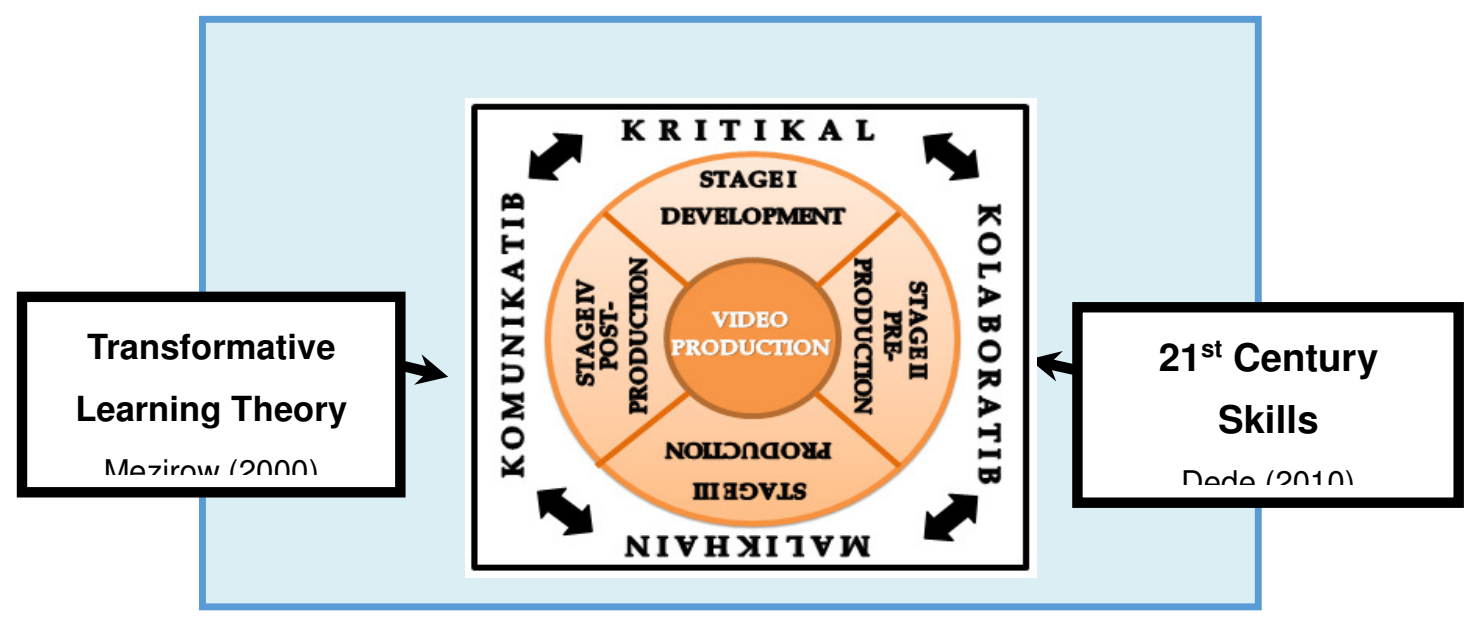

Figura 1. Iskematik Dayagram ng Pag-aaral

Sa ipinahayag nina Dede (2010) ang mga mag-aaral ay kailangang linangin sa digital at sa mga kaalamang kasanayan sa $21^{\text {st }}$ century. Ito ay tungkol sa pagkatuto at integrasyon ng karunungan sa paghahatid sa mga mag-aaral gamit ang teknolohiya, na kung saan ang tumuturo ay tagapagdaloy na lamang. Ang mga gawain ay nakaatang lahat sa mga mag-aaral. Hahayaan ang mga mag-aaral na makatuklas mula sa kanilang mga sarili. Makabuo ng mga konklusyon base sa kanilang mga ginagawa. Sa 21st century, ang mga mag-aaral ang siyang prodyuser o makabuo ng isang makabagong medya hindi sila ang konsyumer. Kung kaya, sa pamamagitan ng video production bilang transpormatibong estratehiya sa pagpapaunlad ng $21^{\text {st }}$ century na kasanayan, titingnan isa-isa ang mga palatakdaan ang kasanayan ng bawat mag-aaral sa tersyarya sa bawat yugto ng video production -ang development, pre-production, production, at post production. Mula rito, bumuo ng isang modelo ang mananaliksik na maglalarawan sa nalinang na kasanayan ng mag-aaral sa terysarya sa bawat yugto ng video production.

\section{Metodolohiya}

\subsection{Disenyo ng Pag-aaral}

Ginamit sa pag-aaral na ito ang descriptive-exploratory design na kung saan inobserbahan ang nalinang na $21^{\text {st }}$ Century Skills ng mga kalahok gamit ang video production bilang transpormatibong estratehiya sa apat na yugto nito (ang development, pre-production, production, at post production). Ang disenyong ito ay isang paraang metodolohikal na naglalayong makatuklas at makabuo ng panibagong kaalaman sa isang partikular na bagay o gawain. Tumutuon ito sa pag-aanalisa sa naobserbahan at sa focused-group discussion upang mailatag ang mga kaalaman sa hinaharap na pag-aaral gamit ang video production bilang transpormatibong estratehiya ng mga mag-aaral at maipaliwanag ang kaalaman sa pamamagitan ng isang kasalukuyang umiiral na teorya.

\subsection{Kalahokng Pag-aaral}

Ang kalahok ng pag-aaral na ito ay mga mag-aaral na nasa sa ikatlong taon sa tersyarya ng Southern Leyte State University - College of Teacher Education na kumukuha ng kursong Bachelor of Secondary Education na nagpapakadalubhasa sa asignaturang Filipino. Binubuo ng 14 na mag-aaral ang kalahok. Ang mga kalahok na 
Galdo, M.

napili ay may parehong kasanayan at lantad sa paggamit ng mga digital na mga kagamitan. Ang 14 na kalahok ay pinangkat ng mananaliksik sa dalawang grupo. Sa paghahati ng mga kalahok, mabusisi at mausisa ang pagpapangkat upang pantay ang distribusyon ng kasanayan ng bawat pangkat.

\subsection{Lugarng Pag-aaral}

Ang pag-aaral na ito ay isinagawa sa pinakatanyag na institusyon sa lalawigan ng Timog Leyte na bumabandera sa Teacher Education ay ang Southern Leyte State University - College of Teacher Education na kung saan mula sa institusyong ito ang mga kalahok sa pag-aaral na ito. Sa kasalukuyan, patuloy na umaangat ang katayuan ng institusyon dahil sa maganda nitong performans sa paghasa sa iba't ibang kasanayan ng bawat mag-aaral na magiging guro. Kilala rin ang paaralang ito sa pagiging produktibo at highly competitive na paaralan. Ang kursong BSED at BEED ang flagship ng institusyon ito. Sa kasalukuyan, patuloy na lumalawak ang paaralan bilang tanda ng kasulungan kaalinsabay ang mga pagbabagong hatid ng siyenya at teknolohiya. Ang kabuuang pahayag ay palatandaan ng pagkatamo sa bisyon-misyong pananaw upang matamo ang pang-akademikong kagalingan sa pamamagitan ng tatlong pangunahing gampanin gaya ng instruksyon, pananaliksik at serbisyo publiko, paghubog ng normal sa sinuman upang makapagbahagi sa inaasahang kaunlaran at kabisaan sa lahat ng klase ng tao..

\subsection{Instrumento ng Pag-aaral}

Sa pag-aaral na ito ay binigyang-diin ng mananaliksik ang mga panukatan gamit ang Observational Checklist sa pagsusuri ng apat (4) na komponents ng $21^{\text {st }}$ Century skills (kritikal, malikhain, kolaborasyon, komunikasyon) ng mga mag-aaral. Mula sa $21^{\text {st }}$ Century Skills Standard Rubrics na nakuha sa www.k12.wa.us/CareerTechEd/.../21stCenturySkillsStandardsRubric.doc ang gagamitin ng mananaliksik. Ito ang nagsilbing batayan ng mga tagasuri/ebalweytor sa pagkilala sa antas ng kasanayan sa $21^{\text {st }}$ Century ng mga mag-aaral sa bawat yugto ng video production.

Ang rubrics ay nahati sa apat na dibisyon, ito ang Creativity and Innovation, Critical Thinking and Problem Solving, Communication, at Collaboration. Bawat dibisyon na ito ay may kaakibat na indikador. Sa Creativity and Innovation may walong (8) indikador, Critical Thinking and Problem Solving may walong (8) indikador, Communication may lima (5) at sa Collaboration naman ay binubuo ng tatlong (3) indikador. Sa pamamagitan ng rubrics na ito, masusukat ang mga nalinang na kasanayan ng mga mag-aaral gamit ang video production bilang transpormatibong estratehiya. Makilala ang nalinang na kasanayan ng mga mag-aaral gamit ang palatakdaan na ito: lampas sa pamantayan (4), tumugon sa pamantayan (3), malapit sa pamantayan (2) at wala sa pamantayan (1).

Sinuri rin ang naitalang fieldnotes $\mathrm{ng}$ mga ebalweytor sa ibaba ng rubrics. Dito isinulat ng tagasuri/ebalweytor ang mga mahalagang detalyeng naobserbahan nila sa naisagawang awtput ng mag-aaral na hindi nakasaad sa rubrics.

\subsection{Paraan ng Pangangalap at Pagsusuri ng datos}

Pagkatapos pahintulutan ang mananaliksik sa kanyang pag-aaral na gagawing kalahok ang napiling mga mag-aaral sa ikatlong taon ng BSED-Filipino, personal na nakipag-usap ang mananaliksik sa labing-apat (14) na kalahok na napili at hinati ang mga kalahok sa dalawang pangkat batay sa antas ng kasanayan nila.

Nang mahati na ang mga kalahok sa dalawang pangkat, nagkaroon ng oryentasyon ang mananaliksik sa ginawang awtput. Inilahad ng mananaliksik ang layunin ng gawain, mekaniks at kriterya sa mga kalahok upang magkakaroon sila ng gabay sa pagbubuo ng video. Inilahad din ang iskedyul ng gawain sa bawat yugto ng video production upang magkaroon ng sistematikong pagsasagawa sa video.

Sa loob ng silid-aralan ginanap ang Development stage at Pre-Production stage samantalang ang Production

4 Consortia Academia Publishing (A partner of Network of Professional Researchers and Educators) 
stage at Post-Production stage ay sa labas ng paaralan batay sa kanilang napiling lokasyon o tagpuan sa nabuong kwento. May kabuuang apat na yugto ang ginawa ng mga kalahok sa pagbuo ng video. Habang nagsasagawa sila ng video, nagkaroon din ng pagmamasid at pagtatasa ang mga nakuhang tatlong tagasuri, kung saan ginawa sa loob ng mahigit dalawang linggo.

Sa bawat yugto na ginawa ng mga kalahok ay sinuri at inoobserbahan ng tatlong (3) tagasuri kasama na ang mananaliksik. Ang dalawang tagasuri na ito ay pawang mga guro sa wika at panitikan sa SLSU-College of Teacher Education na mahusay rin sa larangan ng dula at teatro. Rubrics ang ginamit ng mga tagasuri sa pagkilala sa mga nalinang na kasanayan ng $21^{\text {st }}$ Century Skills sa Video Production bilang Transpormatibong Estratehiya. Ang rubrics na ito ay hango sa $21^{\text {st }}$ Century Skills Standard Rubrics mula sa www.k12.wa.us/CareerTechEd/.../21stCenturySkillsStandardsRubric.doc.

Mula sa nagawang pagtatasa ng tagasuri, nagkakaroon ng focus-grouped discussion ang mananaliksik sa mga kalahok sa mga nakuhang datos sa observational checklist at field notes ng mga tagasuri. Dito ilalahad at iberipika ng mananaliksik ang mga natuklasang datos sa ginawang awtput kung may kulang o pagdadagdag sa natuklasan. Isang kaparaanan ito upang maiwasto ang pananaw ng mga tagasuri sa pananaw naman ng mga kalahok.

\section{Resulta at Diskusyon}

Inilahad sa modelo ang nalinang na $21^{\text {st }}$ century skills ng mga mag-aaral sa video production bilang transpormatibong estratehiya at pagbigay-pakahulugan sa mga datos.

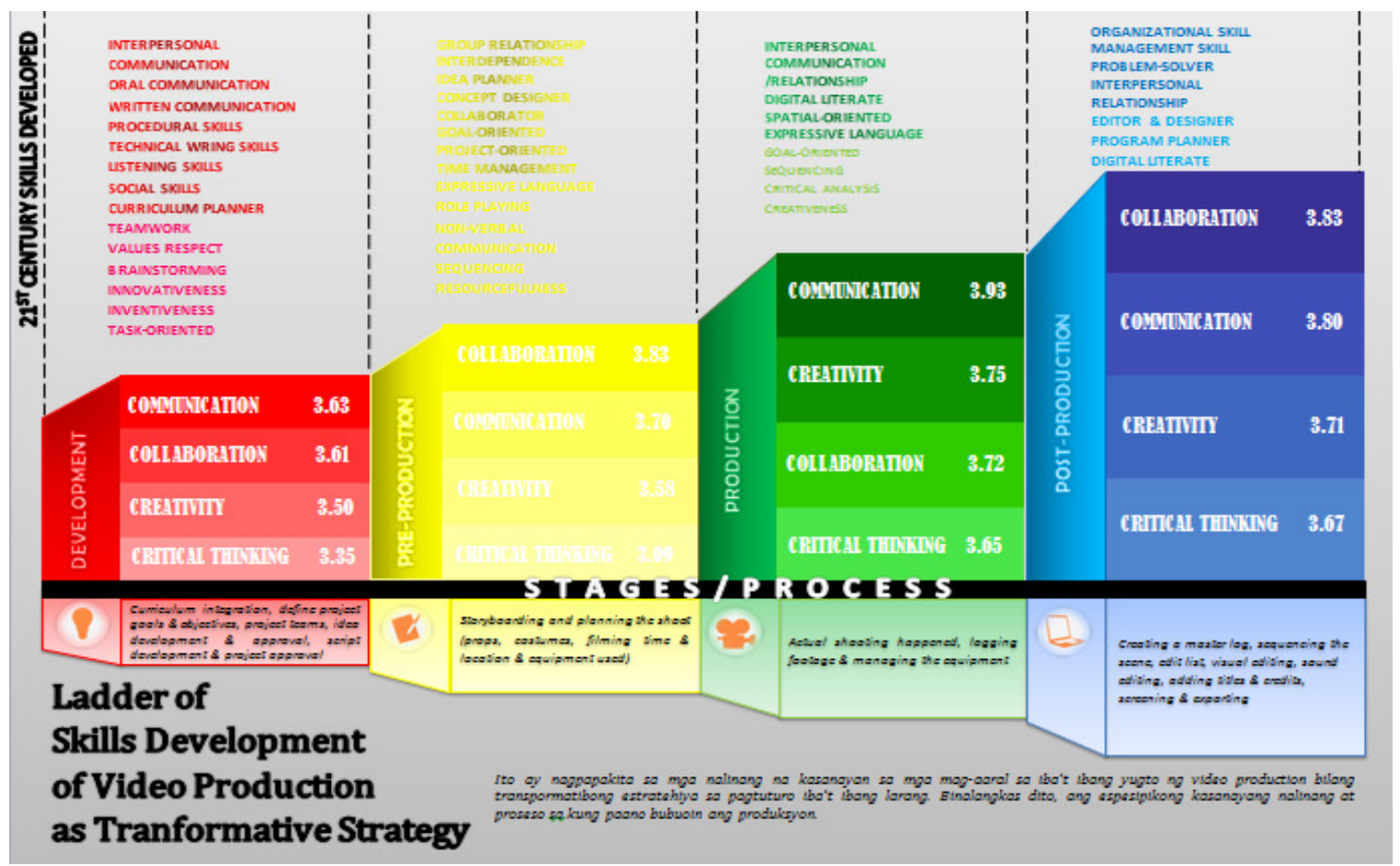

\section{Development Stage}

Ang Development Stage ay unang yugto ng video production na pinalooban ng mga pangunahing hakbang ayon sa sumusunod: Curriculum Integration, Define Project Goals and Objectives, Project Teams, Idea Development and Approval, Script Development at Project Approval.

Inilahad dito na ang apat na C's (communication, collaboration, creativity \& critical thinking) ay nasa lampas sa pamantayan. Ngunit higit na naipamalas ng mga mag-aaral sa yugtong ito ang kasanayang komunikatibo (communication) na may 3.63 at kolaboratibo (collaboration) na may 3.61 na parehong mataas na 
Galdo, M.

mean. Sinundan ito sa kasanayang malikhain (creativity) na may average mean 3.50 at 3.35 naman ang nakuhang average sa kasanayang kritikal (critical).

Makikita sa modelo ang kabuuang kalabasan ng resulta ng pag-aaral sa nalinang na $21^{\text {st }}$ century skills sa Development stage ay ang kasanayan ng mag-aaral sa interpersonal na komunikasyon (pasalita o pasulat man) kabilang ang feedbacking, listening skills, concept-mapping, technical skills (outlining, writing, editing, revising) at procedural skills. Nagpapahiwatig ito na maraming nalilinang na kasanayan sa Development Stage. Nakitaan na ang mga kalahok ng kahasaan ng kasanayang pangkomunikatibo dahil sa nabigyan nang pagkakataon ang mga miyembro ng pangkat na maibahagi ang ninanais nilang mangyari sa takbo ng kwento. At ito ay napatunayan mula sa pagmamasid ng mga ebalweytor na mas aktibo ang mga mag-aaral sa epektibong komunikasyon.

Nagpapahiwatig din ito na ang kasanayang malikhaing pag-iisip ay pagtuunan ng pansin ng guro. Iminungkahi ni Rodriguez at Perez et al. (2019) na huwag ipagwalang bahala ang kasanayang ito datapwa't bigyang-pokus ito dahil sa pagdebelop ng yugto mas malilinang ang pagkamalikhain ng mag-aaral. Napagtanto ng mananaliksik ang malaking papel at responsibilidad sa adhikain ng guro na armasan ang mag-aaral para maging isang $21^{\text {st }}$ century na mag-aaral. Ang pagpapaigting ng gawain na kasangkot ang pagpapalinang ng kasanayang kritikal na pag-iisip ay kinakailangan. Ang paggawa ng karampatang paghahasa ng isipan ng mga mag-aaral sa gawaing pangklase ay inaasahan upang mas malaliman ang pag-unawa ng mag-aaral sa kanilang kaalaman.

\section{Pre-Production Stage}

Sa yugtong ito, pinoproseso ang storyboarding at planning the shoot. Ang mga hakbang na ito ay mahalaga upang maisakatuparan ang unang yugto. Sinuri ang yugtong mula sa mga hakbang upang matukoy ang nalinang na kasanayan sa 21st century (Pianfetti et al., 2003).

Makikita naman ang resulta ng pagsusuri sa mga nalinang na 21st century skills sa Pre-production stage ng video production. Parehong nakakuha ng lampas sa pamantayan ang apat na C's (creativity, critical thinking, communication, collaboration) ngunit nangunguna sa yugtong ito ang kasanayang kolaboratibo na may mean na 3.83 at sinundan ng kasanayang komunikatibo na may 3.70 na mean, 3.58 naman ang mean sa kasanayang malikhain (creativity) at ang kasanayang kritikal (critical thinking) ay may 3.09 mean.

Sa bahaging ito nangunguna ang kasanayang kolaboratibo at sinundan ng kasanayang komunikatibo. Makikita na hindi lalayo ang resulta ng komunikatibo sa kolaboratibo. Ang dalawang kasanayang ito ay may kaugnayan sa pagpapatakbo ng produksyon.

Sa kabuuang resulta ng mga nalinang na 21st century skills sa Pre-production stage ng video production, ang kasanayang kolaboratibo, komunikatibo at malikhain ang nalinang na kasanayan. Patunay ito na ang kalabasan ng pagkatuto ay ang sumusunod: group relationship, interdependence, idea planner, concept designer, group collaboration, goal oriented, project-oriented and time management. Ang pagpapayaman ng gawaing may koneksyon sa pagpapalinang ng kritikal na pag-iisip ay nararapat na bigyang-halaga sa yugtong ito. Ang kasanayang ito ay kinakailangan ng pagtugon sa gawaing nagpapalawak sa reasoning power ng mag-aaral. Bilang edukador, ang paggamit ng varayti ng gawain sa klase ay kailangan sa pagpapayabong ng reasoning power ng mag-aaral.

\section{Production Stage}

Ang Production Stage ay ikatlong yugto ng video production. Inilahad sa Figura 2 ang resulta ng kasanayang ipinakita ng mga kalahok sa gawaing ito. Pinoproseso sa yugtong ito ang aktwal na shoot. Dito na maisasakatuparan ang naplanong konsepto at ideya sa naunang yugto. Maaring may kakaunting pagbabago

6 Consortia Academia Publishing (A partner of Network of Professional Researchers and Educators) 

ngunit pagbabatayan pa rin ang inisyal na konsepto.

Makikita sa modelo na ang lahat ng 4 C's ay lampas sa pamantayan. Nangunguna ang kasanayang komunikatibo mula sa apat na kasanayan na may mean na 3.93, malikhaing kasanayan (creativity) na may 3.75 na mean. Pangatlo sa ranggo ang kolaboratibong kasanayan na may 3.72 at ang kritikal na kasanayan (critical thinking) ay may mean na 3.65 .

Sandigan ang resultang ito sa pahayag nina Hobbs (2001) na ang production stage ay isang yugto sa pagsagawa ng video na nagbibigay tuon sa pagkilala ng tamang koneksyon ng aktor at awdens sa pagbabahagi ng akmang emosyon, damdamin, at karaterisasyon na makikita sa komunikasyon ng actor.

Ang kalabasan ng resulta sa pag-aaral ng mga nalinang na kasanayan sa production stage ng video production ay ang apat na kasanayan ang komunikasyon, kolaborasyon, malikhaing pag-iisip at kritikal na pag-iisip. Ang kasanayang nalinang sa yugto ito ay ang mga sumusunod: digital literacy, spatial orientation, expressive language (idiomatic expressions) and interpersonal communication. Isang malaking tulong ang yugtong ito sa paglinang ng mag-aaral na magkaroon ng 21st century na kasanayan. Kinakailangan ang patuloy na pagtuklas ng estratehiya at pagpapaunlad ng adhikain ng guro para maging matagumpay ang pagkatuto ng mga mag-aaral sa araw-araw na talakayan. Patunay lamang ito na epektibong transpormatibong estratehiya ang video production para sa milenyal na mag-aaral.

\section{Post-Production Stage}

Ang post-production stage ay ang pangwakas na yugto ng video production. Napapalooban ito ng mga hakbang na: master log (scene shoot), sequencing footage, edit list, visual editing, sound editing, adding titles, screening at exporting. Ito ang aktwal na pagyari ng video kung saan dinudugtong-dugtong ang bawat na-shoot na eksena mula sa ikatlong yugto. Dito rin nabibigyang kulay ang mga eksena at kung papaano ihatid nang maayos ang kwento sa mga target awdens (Huerta, 2015).

Ipinakita sa Figura 2 ang mga nalinang na 21st century skills sa post-production stage ng video production. Makikita na ang apat na kasanayang kolaboratibo, komunikatibo, malikhain at kritikal na pag-iisip ay parehong nalilinang at may deskripsiyong lampas sa pamantayan.

Binahagi rin nina Shewbridge at Berge (2004) na ang mag-aaral ay humahanap ng paraan at teknik sa epektibong komunikasyon upang madaling maunawaan at maiintindihan ng kapwa nila ang mga pag-uusap. At ito ay makikita bahaging post production phase sa pagbubuo ng video. Kaugnay rin dito ang nabasang artikulo sa Creative Skillset 2017 na nakasaad ang kahusayan sa komunikasyon ay kinakailangan sa kabuuang produksyon. Ang kasanayang ito rin ay napakahalaga sa pagpapabuti ng mensahe at tema upang ihatid ang makabuluhang kaalaman. Kaya, ang pagkakaroon nang malinaw na audio, animation at special effects sa video ay malaking papel upang maisakatuparan ang kalinawan ng bawat linya at pahayag ng aktor. Sa kalabasan ng pag-aaral ang mga nalinang na kasanayan sa post-production ay ang mga sumusunod: organizational skills, management skill, problem-solver, interpersonal, editor, designer, program planner at digital literate.

Ayon kay Farisi (2016) sa kasalukuyan, ang inobasyon sa pagtuturo ay kailangan ng bawat guro. Isa sa mga magandang inobasyon na ito ay ang paggamit ng mga teknolohiya sa pagtuturo. Ito naman ay sinuportahan nina Bates at Sangra (2011) na nagsabing ang teknolohiya sa kasalukuyan ay isa sa mga pinamakahalagang pangangailangan ng bawat guro upang makasunod sa tinatawag nilang global digital era. Ang mga e-kagamitan tulad ng mga video clips, movie, at iba pa ay isang magandang inobasyon na makapagpalinang sa apat na aspeto ng 21 st century.

Ipinahayag din nina Hofer at Swan (2005) na tulad ng paggamit ng telebisyon isang magandang kagamitan sa pag-abot ng 21st century. Ang pag-aaral na ito ay kahawig sa natuklasan nina Hathaway at Norton (2012) na ang gawaing pagsasadula ay makalilinang sa kasanayang komunikatibo at kolaboratibo. Hindi magtagumpay ang 
Galdo, M.

isang dula kung walang sapat na kasanayan ang mga nagsisipagdula sa mga ito.

Nangangahulugan lamang na ang video production ay isang magandang inobasyon o estratehiya sa pagpapalinang sa kasanayan ng mga mag-aaral. Ang mga mag-aaral sa kasalukuyan ay nangangailangan ng mga motibasyon upang ang kawilihan ng mga mag-aaral. Sa kasalukuyan isa sa malaking isyu sa pagtuturo ay ang interes ng mga mag-aaral sa talakayan. Sa ilang taong pagtuturo, ang kasanayan ng komunikasyon ay naging suliranin ng guro kung kaya naging gawain sa pagpapalinang nito ang pagsasadula. Natuklasan na sa gawaing ito ay nakatutulong sa pagpapalinang ng kasanayang komunikasyon na kahit hindi masyadong mapansin ang pagkakamali ng kanilang pambalarila pero naoobserbahan nadagdagan ang kanilang tiwala sa sarili.

\section{Konklusyon at Rekomendasyon}

Mula sa pag-aaral na ito, ang video production ay isang transpormatibong estratehiya sa pagpapalinang sa $21^{\text {st }}$ century skills ng mag-aaral sa tersyarya partikular sa kasanayang komunikatibo at kolaboratibo. Natuklasan na ang kasanayang komunikatibo ay pinakanalinang sa una at ikatlong yugto at kolaboratibo ang pinakanalinang sa ikalawa at ikaapat na yugto ng video production. Ang kasanayang kritikal na pag-iisip ay pumapahuli sa bawat yugto. Patunay ito na ang pagtuon ng kasanayang kritikal na pag-iisip ay kinakailangang tugunan ng mga guro sa pagtuturo ng mga mag-aaral sa $21^{\text {st }}$ century gamit ang video production. Ang pagbuo ng mga gawaing angkop sa pagpapalinang ng kritikal na pag-iisip ay inaasahan sa bawat estratehiya ng pagtuturo upang mas handa ang mga mag-aaral sa pagpasok ng henerasyong digital. Dagdag pa, ang paglantad sa mga guro sa mga dulog na magpapaigting sa paghahasa ng isipan ay narapat na kaakibat sa proseso ng pagtuturo-pagkatuto sa bawat guro na sinasabayan ng makabagong pamamaraan sa pagtuturo.

Batay sa resulta ng pagsusuri iminungkahi ng mananaliksik ang mga sumusunod: (a) kinakailangan ang pagpapaigting at ibayong paglinang ng estratehiya ng mga guro upang mahasa at mapalawak ang kaisipan ng mga mag-aaral sa pag-unawa gamit ang makabagong paraan ng pagtuturo; (b) ang guro ay dapat na gumamit ng makabagong paraan ng pagtuturo gaya ng video production upang magkaroon ng transpormasyon sa pagkatuto ng mag-aaral sa ika-21 na siglo; at (c) gamitin ang nabuong awtput ng pag-aaral bilang estratehiya sa pagpapalinang ng kasanayan ng mga mag-aaral sa ika-21 na siglo.

Acknowledgement: Nais kong ipaabot ang aking taos pusong pasasalamat sa lupon ng tagasuri sa pag-aaral na ito, sa aking tagapayo, sa mga mag-aaral ko sa nasabing institusyon sa patuloy na pagsuporta na maging isa sa mga kalahok ng pag-aaral na ito, at sa lahat ng mga tao na nagsilbing inspirasyon at motibasyon sa katagumpayan nito.

\section{Sanggunian}

Alvermann, D. E. (2002). Adolescents and literacies in digital world. New York: Peter Lang.

Bates, A., \& Sangra, A. (2011). Managing technology in higher education: Strategies for transforming teaching and learning. San Francisco, CA: Jossey-Bass.

Bull, G L., \& Bell, L. (2010). Teaching with digital video: watch, analyze, create. Washington, DC: International Society for Technology in Education.

Cayari, C. (2015). Participatory culture and informal music learning through video creation in the curriculum. International Journal of Community Music, 8 (1), 41-57. https://doi.org/10.1386/ijcm.8.1.41_1

Dede, C. (2010). Comparing frameworks for 21 st century skills. In J. Bellanca \& R. Brandt (Eds.) 21st Century Skills (pp. 51-76). Bloomington, IN: Solution Tree Press.

Farisi, M. I. (2016). Developing the 21st-century social studies skills through technology integration. Turkish Online Journal of Distance Education, 17, 16-30.

Hathaway, D., \& Norton, P. (2012). Video production and classroom instruction: Bridging the academies and the realities of practice in teacher education. Journal of Technology and Teacher Education, 20(2), 127-149.

Hobbs, R. (2001). The great debates circa 2001: The promise and the potential of media literacy. Community

8 Consortia Academia Publishing (A partner of Network of Professional Researchers and Educators) 
Media Review, 24(1), 25-27.

Hofer, M., \& Swan, K. (2005). Digital moviemaking-the harmonization of technology, pedagogy and content. International Journal of Technology in Teaching and Learning, 1(2), 102-110.

Hoffenberg, H., \& Handler, M. (2001). Digital video goes to school. Learning and Leading with Technology, 29(2), $10-15$.

Huerta, M. (2015). Through the lens of film-making. Edutopia. https://www.edutopia.org/blog/through-the-lens-of-filmmaking-merle-huerta

Jenkins, H. (2009). Confronting the challenges of participatory culture: Media education for the 21 st century. (The John D. and Catherine T. MacArthur Foundation Reports on Digital Media and Learning) Cambridge, MA: MIT press.

Kearney, M., \& Schuck, S. (2006). Spotlight on authentic learning: Student developed digital video projects. Australasian Journal of Educational Technology, 22(2), 189-208.

Mezirow J. (2000). Transformative learning: Theory to practice. In P. Cranton (Ed.). Transformative learning in action: Insights from practice (pp. 5-12). New directions for adult and continuing education. Jossey-Bass.

Norton, P., \& Hathaway, D. (2010). Video production as an instructional strategy: Content learning and teacher practice. Contemporary Issues in Technology and Teacher Education, 10(1), 145-166.

Pianfetti, E. S. (2001). Focus on research: teachers and technology: Digital literacy through professional development. Lang. Arts, 78(3), 255-62.

Rodríguez, G., Pérez, N., Núñez, G. et al. (2019). Developing creative and research skills through an open and interprofessional inquiry-based learning course. BMC Med Educ, 19, 134. https://doi.org/10.1186/s12909-019-1563-5

Shewbridge, W., \& Berge, Z. (2004). The role of theory and technology in learning video production: The challenge of change. International Journal on E-Learning, 3(1), 31-39.

Snelson, C. (2018). Video production in content-area pedagogy: a scoping study of the research literature. Learning, Media and Technology, 43(3). https://doi.org/10.1080/17439884.2018.1504788 
Galdo, M.

10 Consortia Academia Publishing (A partner of Network of Professional Researchers and Educators) 